RECONSTRUYENDO EL CONOCIMIENTO DIDÁCTICO DE PROFESORES UNIVERSITARIOS DE LA UNIVERSIDAD NACIONAL DEL Nordeste. Páginas 131-148 en Revista de la Escuela de Ciencias de la Educación, año 11, número 10, enero a DICIEMBRE DE 2015. ISSN 1851-6297. ISSN EN LINEA 2362-3349.

\title{
RECONSTRUYENDO EL CONOCIMIENTO DIDÁCTICO DE PROFESORES UNIVERSITARIOS DE LA UNIVERSIDAD NACIONAL DEL NORDESTE
}

\author{
Por Patricia B. Demuth* \\ Universidad Nacional del Nordeste, Argentina. \\ patriciademuth@hotmail.com
}

\section{Resumen}

En el artículo se presenta el análisis de un caso incrustado de 11 profesores universitarios de la Universidad Nacional del Nordeste - Argentina, a través de la metodología narrativa.

Se abordan temáticas vinculadas al conocimiento didáctico del contenido de los profesores, su relación con los contextos académicos, profesionales y disciplinares, y las diferencias que se evidencian entre docentes experimentados y docentes principiantes afines a cuatro asignaturas de carreras variadas: Odontología, Informática, Ciencias de la Educación e Historia.

\section{Palabras clave:}

Conocimiento didáctico del contenido - Docencia universitaria - Identidad docente.

\section{Abstract:}

In this paper we present an analysis of a case embedded of 11 university teachers from Universidad Nacional del Nordeste - Argentina, through the narrative methodology.

It addresses topics related to pedagogical content knowledge of teachers, their relationship with the academic, professional and disciplinary contexts, and show the differences between experienced teachers and beginning teachers related to four subjects varied careers: Dentistry, Computer Science, Pedagogy and History.

\section{Key Words:}

Pedagogical content knowledge - University teachers - Teacher identity.

* Prof. y Lic. en Ciencias de la Educación (Universidad Nacional del Nordeste - UNNE); Especialista en Ciencias Sociales con mención en Curriculum y Prácticas Escolares (FLACSO); Doctora en Didáctica y Organización de Instituciones Educativas (Universidad de Sevilla). Profesora Titular Ordinaria del Espacio Integrador "Pedagogía Universitaria y Tecnología Educativa" (Facultad de Medicina de la UNNE) y Auxiliar Docente Ordinaria de Primera Categoría en la asignatura Didáctica I/Didáctica General de Facultad de Humanidades de la UNNE. 
Revista de la Escuela de Ciencias de la Educación, año 11, número 10, enero a diciembre de 2015. Páginas 131-148. ISSN 1851-6297. ISSN EN LINEA 2362-3349. RECONSTRUYENDO EL CONOCIMIENTO DIDÁCTICO DE PROFESORES UNIVERSITARIOS de la Universidad Nacional del Nordeste. Patricia B. Demuth

\section{Introducción}

El artículo que vamos a desarrollar presenta desafíos particulares en su redacción, estructuración y posterior lectura, ya que el formato asumido es una trama narrativa de un caso incrustado (Yin, 1994). Dicha trama narrativa se ha ido tejiendo con las voces de los actores protagonistas del caso, los actores que operan como referentes teóricos y metodológicos, y la voz de quien decide reunirlos en esta singular forma de construcción. Lo que aquí se presentará forma parte de un trabajo mayor en el marco de un Programa de Doctorado en Didáctica y Organización de Instituciones Educativas de la Universidad de Sevilla.

Los protagonistas del caso son profesores universitarios en ejercicio pertenecientes a diferentes carreras universitarias que se dictan en la Universidad Nacional del Nordeste, en adelante UNNE. Estas son: Odontología, Licenciatura y Profesorado en Historia, Licenciatura y Profesorado en Ciencias de la Educación y Licenciatura en Sistemas de la Información. La narrativa del caso ha tenido como objetivo el desentrañar los procesos de construcción del conocimiento didáctico de dichos profesores que ejercen la docencia desde diferentes formaciones, experiencias y roles.

Entendemos que la narrativa es una herramienta que utiliza el investigador junto con los sujetos investigados para construir una realidad determinada, para construir mundos posibles que permitan ajustar la mirada, vincular tradiciones, culturas, vivencias. Una construcción unificada de diferentes relatos, mezclados con asertos y reflexiones sobre la temática escogida.

Una construcción única e irrepetible de la vivencia desarrollada "en" y "sobre" lo investigado, y con la marca y estilo personal de quienes la redactan; en la que se explicitan aquellos componentes subjetivos que la conforman, no sólo reconociéndolos sino que también aceptándolos como valiosas oportunidades de reflexión, ya que las impresiones del investigador se incorporan al relato.

Luego del análisis de los discursos, de su interpretación y asertos parciales, la narrativa se convierte en el proceso recursivo que vuelve a trabajar las ideas más importantes permitiendo una mayor profundización en la comprensión. Se mueve entre el pasado y el presente, recordando momentos y pareceres, pero también generando nuevas interpretaciones y relaciones.

Su elaboración ha implicado la definición "un sistema delimitado en tiempo y espacio de actores, relaciones e instituciones sociales" (Neiman y Quaranta, 2006 , p. 220). En una dirección similar junto con Stake (2007, p.16) sostenemos que este caso narrado es algo específico, algo complejo, en funcionamiento, es algo sobre lo que se ha buscado profundizar, desentrañando su singularidad, buscando comprender, interpretando.

En este sentido, la comprensión y la interpretación son las dos habilidades, los dos "movimientos" que se encuentran en el corazón de esta metodología y que pone en funcionamiento el investigador y el investigado al estudiar el 
Revista de la Escuela de Ciencias de la Educación, año 11, número 10, enero a diciembre de 2015. Páginas 131-148. ISSN 1851-6297. ISSN EN LINEA 2362-3349. RECONSTRUYENDO EL CONOCIMIENTO DIDÁCTICO DE PROFESORES UNIVERSITARIOS de la Universidad Nacional del Nordeste. Patricia B. Demuth

problema u objeto de investigación. Objeto muy especial, nos va a afirmar, Rodríguez Rojo (2000), "tan especiales que, en muchas ocasiones, como en ésta, no son objetos sino sujetos. La naturaleza de la investigación se concreta en problemas humanos" (Rodríguez Rojo; 2000, p.120).

Teniendo en cuenta lo anterior consideramos que el caso que narraremos aborda

- un proceso: el de la construcción del conocimiento didáctico del contenido de profesores universitarios miembros de los Departamentos de Informática, Odontología Rehabilitadora, Historia y Ciencias de la Educación de la UNNE

- con un alcance específico desde el que se pretende iniciar la comprensión del fenómeno en dicha institución universitaria

- su naturaleza es considerada típica en el contexto en la que se identifica, los equipos de profesores participan de las actividades académicas habituales en la institución y no presentan notas distintivas, salvo la de pertenecer a colectivos determinados que centran nuestro interés

- es un caso contemporáneo ya que los profesores iniciaron sus actividades hace no más de 25 años en algunos casos y 5 años en otros, y continúan ejerciendo en los mismos cargos al momento de esta presentación

- desde esta clasificación lo ubicamos como caso múltiple incrustado, ya que hacia el interior del mismo encontramos configuraciones de acuerdo a la pertenencia a los departamentos/asignaturas $\mathrm{y}$, a su vez, diferentes unidades de análisis: cada uno de los profesores en estudio. Si bien el gran contexto es la Universidad Nacional del Nordeste, los contextos concretos son cada uno de los departamentos de pertenencia.

- su uso poseerá las dos características, será exploratorio en un primer momento e intentará avanzar hacia el análisis (e inclusive la explicación como veremos más abajo) dentro de un enfoque meramente cualitativo.

Por otro lado, haciendo hincapié en la importancia de la validez y la confiabilidad del proceso (Jiménez Chaves, 2012, p.148), resaltamos la triangulación de instrumentos y fuentes de recogida de la información: se realizaron observaciones no participantes de clases, entrevistas y análisis de documentos personales y curriculares durante todo un período de dictado de las asignaturas. Esta triangulación desde diferentes instrumentos de recogida permite que la información obtenida sea considerada como tipos de "discursos" que se reconstruirán a través de la estrategia narrativa.

Si bien es cierto que se puede considerar también a la narrativa como un método de recogida de la información (Bolívar, 2002), debido a que en este 
Revista de la Escuela de Ciencias de la Educación, año 11, número 10, enero a diciembre de 2015. Páginas 131-148. ISSN 1851-6297. ISSN EN LINEA 2362-3349. RECONSTRUYENDO EL CONOCIMIENTO DIDÁCTICO DE PROFESORES UNIVERSITARIOS de la Universidad Nacional del Nordeste. Patricia B. Demuth

trabajo no se la desarrolla como historia de vida, si no que los instrumentos mencionados guían el relato hacia los aspectos de interés, hemos decido emplearla como estrategia de análisis y redacción, posterior a las entrevistas y a la recolección de los instrumentos.

Dadas las recomendaciones que realizan los especialistas en esta metodología, realizamos un relato no muy extenso en el que se sintetizan aquellos aspectos que consideramos más importantes del caso colectivo con casos incrustados, intentando encontrar puntos de acercamiento y respetando las diferencias más notables. Los dejamos con el relato del caso...

\section{Los profesores de la UNNE: la docencia universitaria entre la investigación científica y la profesión independiente}

Comenzar el relato implica intentar 're-ingresar' nosotros y 'hacer ingresar' al lector al múltiple universo que constituye el mundo universitario de la UNNE, a sus variados colectivos académicos que la integran, distribuidos en dos grandes sedes asentadas en dos provincias argentinas: Chaco y Corrientes. Ingresar a ese complejo de relaciones interpersonales e institucionales que se deben conocer, nos traslada al pasado y al mismo proceso de selección de los sujetos con los cuales investigar, definiendo quiénes iban a constituirse en los casos incrustados del caso colectivo.

En un primer momento, el contacto con informantes clave nos dio datos sumamente valiosos para nuestra investigación relacionados con posibles candidatos que podían llegar a cumplir con nuestros criterios de selección de la muestra, y estarían abiertos a participar de una experiencia como ésta.

Habíamos observado que necesitábamos conocer y comprender lo que podría estar sucediendo en diferentes contextos académicos y con profesores con experiencias también variadas. Queríamos entonces, trabajar con equipos docentes de asignaturas de diferentes campos del conocimiento que tengan entre sus miembros a principiantes y experimentados. Entendiendo a los primeros como profesores con menos de 5 años de trayectoria en docencia universitaria, y a los segundos con más de 15 años en la carrera docente.

Fue así que los informantes de las diferentes facultades nos facilitaron un número muy amplio de correos electrónicos para comenzar a contactarlos.

El primer contacto lo realizamos con los responsables de las asignaturas que tenían en su equipo docente a principiantes. Por las características de la carrera universitaria, los responsables de asignaturas son todos profesores experimentados. Una vez que ellos dijeron que sí, nos escribimos con los profesores principiantes para conocer de su interés en participar, necesitábamos que todos estén de acuerdo.

Terminamos de definir la muestra cuando consideramos tener el sí de un colectivo profesional lo suficientemente variado disciplinar y pedagógicamente hablando. 
Revista de la Escuela de Ciencias de la Educación, año 11, número 10, enero a diciembre de 2015. Páginas 131-148. ISSN 1851-6297. ISSN EN LINEA 2362-3349. RECONSTRUYENDO EL CONOCIMIENTO DIDÁCTICO DE PROFESORES UNIVERSITARIOS de la Universidad Nacional del Nordeste. Patricia B. Demuth

La misma se cerró con cuatro casos incrustados: Informática: con una profesora experimentada y dos principiantes; Odontología: con una profesora muy experimentada, dos experimentados de mediana antigüedad y una principiante; Historia: con una profesora experimentada y un principiante; y Ciencias de la Educación: con una profesora muy experimentada y una principiante. Cuatro equipos docentes pertenecientes a departamentos reconocidos en la universidad por su desarrollo en el plano profesional, de investigación o docente.

Una vez obtenidos todos los accesos iniciamos la tarea de campo.

Las idas y venidas de los diferentes contextos institucionales, con características variadas en relación con sus constituciones edilicias; su mayor, menor o escasa privacidad de acuerdo al caso; las entrevistas en las oficinas de los profesores, las filmaciones en las aulas o en la clínica, las mañanas bien tempranas y las tardes cercanas a las noches, formaron parte de un movimiento intenso en los once meses de recogida de la información.

Grata sorpresa en todos los casos: apertura para colaborar en la investigación y compartir lo que sabían y hacían. Los informantes clave fueron muy certeros en sus recomendaciones.

La primera aproximación en la totalidad de los casos, después del correo electrónico de aceptación, fue a través de sus curricula vitae. Nos pusimos a leerlos, intentado conocerlos un poco, imaginándonos sus perfiles, de acuerdo a sus edades, a los variados antecedentes en docencia, en investigación, en gestión y en la profesión por fuera de la universidad. Los estudiamos en detalle para estar más preparados para nuestra primera entrevista cara a cara.

Concertamos fechas y horas para los encuentros, y allí estuvimos, prontos a la tarea de iniciar el estudio de un caso, de permitirnos indagar en su singularidad, en su particularidad, en su idiosincrasia (Stake, 2007), y para ver si era posible trazar puentes que acerquen contextos aparentemente lejanos para construir la dimensión colectiva de este grupo de profesores.

Comenzamos con las presentaciones de lo que implicaban las entrevistas sucesivas, las observaciones de clase, los materiales que íbamos a necesitar, la necesidad de la grabación digital de lo conversado, y grabación audiovisual de las clases, y del modo de trabajo en general.

Trabajamos entonces, con estos 11 docentes: tres Expertos en Sistemas o Licenciados en Sistemas de la Información, cuatro Odontólogos, dos Profesores y Licenciados en Historia y dos Profesoras en Ciencias de la Educación.

El promedio general de la edad de los principiantes fue de 28 años, el de los profesores de experiencia media de 41 años y el de las profesoras de mayor experiencia de 62 años. Estas cifras nos parecen sumamente ilustrativas y que junto con la antigüedad vinculada a estas edades, nos da cuenta del paisaje complejo y variado al cual nos acercábamos.

Una a una se fueron sucediendo las entrevistas y las observaciones, y nos fueron mostrando realidades diferentes y compartidas, tanto al interior 
Revista de la Escuela de Ciencias de la Educación, año 11, número 10, enero a diciembre de 2015. Páginas 131-148. ISSN 1851-6297. ISSN EN LINEA 2362-3349. RECONSTRUYENDO EL CONOCIMIENTO DIDÁCTICO DE PROFESORES UNIVERSITARIOS de la Universidad Nacional del Nordeste. Patricia B. Demuth

del caso incrustado como en relación con el caso colectivo. De acuerdo con la personalidad de cada uno algunos relatos eran más detallados que otros, más abundantes que otros; pero, sin embargo, al momento de observar las conexiones entre éstos, podíamos darnos cuenta que efectivamente constituían un caso colectivo, en el que compartían pareceres, creencias, conocimientos y actuaciones sobre la circunstancias más concretas y más generales de su quehacer en el contexto universitario. Realmente, el trabajo con los once sujetos nos resultó distendido, agradable y desafiante.

En la reconstrucción de estos relatos, para la construcción de un relato unificado, en el intento de comprenderlos, regresamos a las preguntas iniciales del trabajo de investigación que guió el proceso, y son ellas las que orientarán las dos secciones que continúan. Avancemos.

¿Cómo construyen su conocimiento didáctico del contenido los docentes en estudio? ¿Cuáles son sus fuentes principales? ¿Qué diferencias se pueden observar entre los principiantes y los más experimentados?

Estas tres preguntas sintetizan los puntos más interesantes que intentaremos responder en esta primera parte, yendo de las cuestiones comunes a las particularidades (la variable antigüedad y el campo disciplinar en el que se inscriben).

Los procesos de construcción del conocimiento docente $(C D)$ de los profesores en estudio están constituidos por una red de relaciones singulares en un escenario específico: cada uno de los departamentos, y negociado dentro de los límites de una determinada comunidad guía: la profesional (Cubero, 2005) en el caso de informática y odontología; la académica en el caso de historia; y la académica y la docente en el caso de ciencias de la educación.

Tanto experimentados como principiantes interpretan a través de estos ámbitos, y no de otros, las diferentes informaciones que les rodean, y dilucidando también aquéllas que vivencian en el ejercicio de la docencia, dotándolas de significado, y constituyéndolas en conocimientos prácticos de su accionar (Schön, 1992).

La similitud de los conocimientos prácticos de los sujetos en cada caso incrustado, nos indicaba la presencia de colectivos particulares (Fourez, 2008), que tenían en común cierta orientación hacia alguna o algunas de las dimensiones estudiadas: la docente, la investigación, la gestión o la profesión independiente (por fuera de la universidad). En ninguno de los casos incrustados pudimos observar diferencias entre sus miembros en este aspecto. Como tampoco encontramos presencia de la "Gestión" como dimensión influyente del $C D$ de los profesores.

La complejidad de los procesos de construcción del CD se potencia al indagar sus componentes, todos inter-relacionados, inter-referenciados, en transformación constante, tanto en principiantes como en experimentados. Aún 
Revista de la Escuela de Ciencias de la Educación, año 11, número 10, enero a diciembre de 2015. Páginas 131-148. ISSN 1851-6297. ISSN EN LINEA 2362-3349. RECONSTRUYENDO EL CONOCIMIENTO DIDÁCTICO DE PROFESORES UNIVERSITARIOS de la Universidad Nacional del Nordeste. Patricia B. Demuth

estos últimos reconocen que día a día siguen construyendo y reconstruyendo sus conocimientos docentes en torno a la asignatura y a sus estudiantes.

Lo que sí es notable, es la diferencia en la frecuencia y magnitud de estos cambios en los principiantes, ya que se suceden con mayor regularidad que en los otros momentos profesionales, teniendo en cuenta que el conocimiento docente de estos profesores está en sus inicios (Grossman, 1990).

En líneas generales, los principiantes comparten componentes del CD con los profesores más experimentados con los cuales trabajan. Cuestión que puede interpretarse desde las relaciones de "discípulos-maestros" que se vivencian en el contexto universitario; y de las exigencias institucionales que desarrollan algunas facultades sobre sus miembros.

Respecto de los componentes compartidos, todos los profesores compartían con su equipo docente la formación académica disciplinar, los materiales y, obviamente, el contexto institucional. En los casos de historia y ciencias de la educación compartían también, la formación pedagógica y de investigación; y en el caso de informática y odontología compartían las trayectorias y desempeños profesionales por fuera de la universidad.

Por ello, consideramos que los equipos de las asignaturas, junto con los profesores responsables de mayor experiencia, se constituyen en los principales referentes para el desarrollo de determinados conocimientos, determinadas concepciones y prácticas docentes.

Estas primeras características generales van dando forma al edredón metafórico del que hablaba Becher (2001) y que nosotros asimilamos al conocimiento docente.

En este intento por comprender su forma y contenido mencionamos dos cuestiones que nos resultaron altamente reiterativas en los discursos de todo este colectivo de profesores, la primera tiene que ver con ese genuino interés que manifestaron por sus estudiantes, por los procesos de aprendizaje que ellos realizaban, por sus preferencias al momento de plantear un contenido, por sus fracasos y los diferentes modos de tutoría o apoyo que podrían ayudarles.

Interés siempre presente, tanto en principiantes como experimentados, donde la diferencia se basa en las fuentes que utilizan para el reconocimiento de esos 'otros', ya que pudimos apreciar cómo los principiantes trabajan mayoritariamente desde sus concepciones construidas como estudiantes que fueron, y los profesores con más experiencia trabajan desde conocimientos sólidamente construidos desde su propia experiencia a través de los años como docentes de dicho estudiantado.

De todas formas, en los principiantes reconocíamos que esas concepciones están en proceso de transformarse en conocimientos sobre el aprendizaje de sus estudiantes, o lo que en palabras de Elbaz (1983) significa la construcción de uno de los componentes constitutivos del conocimiento práctico: la experiencia que tiene el docente sobre los estilos de aprendizaje de los estudiantes. 
Revista de la Escuela de Ciencias de la Educación, año 11, número 10, enero a diciembre de 2015. Páginas 131-148. ISSN 1851-6297. ISSN EN LINEA 2362-3349. RECONSTRUYENDO EL CONOCIMIENTO DIDÁCTICO DE PROFESORES UNIVERSITARIOS de la Universidad Nacional del Nordeste. Patricia B. Demuth

En líneas generales y más allá de las fuentes mencionadas, esas experiencias que tanto les interesaba, sobre las que reflexionaban y ajustaban sus propuestas, los acerca, para nuestro modo de ver, a los profesores exitosos de Bain (2007) y Cid-Sabucedo et al (2009); al identificarlos con esa característica común: su dedicación a los estudiantes.

La segunda cuestión que no podemos dejar de mencionar, tiene que ver con las diferencias que existen en las dimensiones mencionadas de acuerdo al caso: sea la profesional, la de investigación, o la que conjuga docencia e investigación.

Nos damos cuenta, que más allá de las metas y objetivos de aprendizaje particulares de las asignaturas, que cada uno de los profesores manifestaron tener muy en claro; lo que realmente estaría funcionando como marco organizador, o mapa conceptual común, para la toma de decisiones sobre la enseñanza (Grossman, 1990) serían las demandas de la Cultura Profesional (del contexto laboral local), las demandas de la Cultura Académica (la perspectiva de conocimiento disciplinar desde la cual se lee y construye el mundo), o las demandas de la Cultura Docente (perspectivas pedagógicas concretas).

En los diferentes casos estos entornos aparecen profundamente conocidos por los experimentados y en menor medida, pero también conocidos, por los principiantes. Entornos en los cuales están insertos, contextos que se constituyen, a la vez, en meta y fuente de conocimiento. En este caso el potencial del curriculum (Gudmundsdótir y Shulman, 1990) estaría desarrollado no sólo por la profundidad y organización del conocimiento de la materia sino por la profundidad en el conocimiento y la experiencia profesional, de investigación y docente. Cuanta más experiencia profesional compartían en el aula, mejores profesores eran, según las reflexiones de los odontólogos e informáticos. Cuanta más experiencia de investigación traducían en estrategias didácticas, mejores procesos de enseñanza y aprendizaje se desarrollaban en las clases de historia. Y en similar línea a los de historia, pero sumando las perspectivas pedagógicas, las docentes de ciencias de la educación.

Siguiendo con la narración, nos dimos cuenta que con el estudio de los diferentes casos estábamos ampliando nuestra mirada tradicional sobre el potencial del curriculum que nos refería al conocimiento de la materia, y sostenía que un conocimiento más elaborado de las estructuras sustantivas y sintácticas de la disciplina estaría indicando posiblemente una mayor eficacia en la docencia. Este conocimiento más elaborado de las estructuras de las disciplinas las pudimos observar en los profesores con más antigüedad en la docencia y con una mayor formación de posgrado.

Hemos observado cómo en los principiantes había sólo un desarrollo incipiente de las mismas, ya que no fueron construidas acabadamente en su formación de grado, como se ha comprobado que mayoritariamente sucede (Grossman,1990), sino que se estaba construyendo recientemente a partir de 
Revista de la Escuela de Ciencias de la Educación, año 11, número 10, enero a diciembre de 2015. Páginas 131-148. ISSN 1851-6297. ISSN EN LINEA 2362-3349. RECONSTRUYENDO EL CONOCIMIENTO DIDÁCTICO DE PROFESORES UNIVERSITARIOS de la Universidad Nacional del Nordeste. Patricia B. Demuth

sus inicios en la formación de posgrado, desde la cual se han ido replanteando conocimientos de manera individual, y también, han presenciado cambios en la organización de los programas de las asignaturas.

Por lo antes dicho, pudimos observar que los conocimientos y concepciones que los profesores experimentados manifestaban sobre la buena enseñanza, se sustentaban en mejores y más profundos desarrollos de conocimientos sustantivos o sintácticos. Mientras que en los principiantes, el conocimiento de la disciplina, se sustentaba más en la "buena práctica" de la misma, en su "buena ejecución" concreta.

Suponemos que esta situación está estrechamente vinculada con las características disciplinares complejas que las constituyen y potenciada aún más, debido a la asignación de los trabajos prácticos a los que los principiantes estaban destinados mayoritariamente.

A partir de los relatos que los profesores iban haciendo, y de estas consideraciones sobre el conocimiento de la materia, nos preguntábamos sobre cómo realizaban las transformaciones del conocimiento disciplinar (fundamental en el proceso), a construcciones didácticamente enseñables, cómo eran estas representaciones didácticas del conocimiento disciplinar (Bolívar, 2005; Marcelo, 2006); y fueron los relatos sobre diferentes contenidos de los programas de las asignaturas, sus detalles más o menos precisos, sus genuinas justificaciones en torno a la búsqueda de un mejor aprendizaje, los que guiaron y nos mostraron similitudes y diferencias.

En primer lugar, todos los profesores, realizaron una valoración altamente positiva del aprendizaje autónomo, valoración que condicionaba la elaboración de estrategias orientadas a propuestas didácticas afines. Frente a estas propuestas el rol docente adquiría un lugar secundario, de guía, de soporte.

Sólo en el caso de odontología, por las características mismas de los conocimientos y sujetos que se ponen en juego en las clínicas, observamos que los profesores asumían, en algunas circunstancias, roles más directivos en sus intervenciones.

Este aprendizaje autónomo se justificaba, una vez más y de acuerdo al caso, desde la cultura profesional o cultura académica, que constantemente lo estaría demandado. De esta forma, en el caso de Informática, por ejemplo, se planteaban simulaciones de situaciones y prácticas profesionales, en las que los estudiantes eran protagonistas y los profesores guías, en ocasiones "socráticos" que buscaban la reflexión de los estudiantes sobre sus propias acciones y en ocasiones "clientes" que evaluaban y corregían lo producido, y en última instancia, cuando no se había logrado el aprendizaje con las estrategias anteriores, se convertían en "explicadores", utilizando analogías y ejemplos prácticos, ya sea con paradigmas previos aprendidos en asignaturas anteriores, o con softwares concretos realizados con esos paradigmas que estaban trabajando. 
Revista de la Escuela de Ciencias de la Educación, año 11, número 10, enero a diciembre de 2015. Páginas 131-148. ISSN 1851-6297. ISSN EN LINEA 2362-3349. RECONSTRUYENDO EL CONOCIMIENTO DIDÁCTICO DE PROFESORES UNIVERSITARIOS de la Universidad Nacional del Nordeste. Patricia B. Demuth

En el caso de Odontología la práctica profesional era directa, en las clínicas trabajaban con pacientes reales en donde los estudiantes deben asumir su rol profesional, y los profesores también tutorizaban, guiaban o intervenían de un modo directivo de acuerdo con las circunstancias.

En el caso de Historia y Ciencias de la Educación se simulaban procesos de reflexión y análisis propios del trabajo de investigación del historiador o de las actividades de investigación/intervención del asesor institucional (perfil profesional de Ciencias de la Educación).

Por lo tanto, el CDC de nuestros profesores con mayor experiencia tenían aspectos disciplinares de mayor complejidad y poseían tres componentes contextuales que lo singularizaban: el del contexto profesional o académico o docente (como ya se indicó), el contexto educativo específico del cual aprendieron durante los años de experiencia, y el contexto universitario más amplio del que eran conocedores desarrollados y en algunos casos referentes nacionales activos.

Mientras que el CDC de los profesores principiantes tenía también aspectos disciplinares que lo constituían pero de menor complejidad y poseían dos componentes contextuales que lo singularizaban: el del contexto profesional o académico o docente (como ya se indicó) y el contexto educativo específico del cual aprendían informalmente sobre la docencia.

Pasaremos a reconstruir estos procesos de aprendizaje de la docencia, presente en todos los docentes, desde la actualidad de los principiantes, y como un recuerdo de su inserción docente en los experimentados.

Podemos caracterizar que todos los profesores en sus relatos referenciaban siempre a otros, a otros cercanos y lejanos, pasibles de ser imitados u olvidados, docentes de la misma asignatura, mentores, o profesores de otras asignaturas, hasta mencionaban a colegas del campo profesional que opinaban y evaluaban los conocimientos de sus estudiantes. Esos otros, que se reiteraban en nombres o anécdotas en cada sujeto, nos permitió observar que conformaban en los principiantes; o conformaron anteriormente, en el caso de los experimentados; contextos más que influyentes sobre los profesores al momento de tomar decisiones curriculares sobre la enseñanza y el aprendizaje de la asignatura, al "delinear", su propio conocimiento didáctico del contenido.

En el caso de los profesores experimentados estos 'otros' influyentes se fueron modificando y ampliando hacia colegas o referentes disciplinares nacionales o internacionales que en la actualidad ejercen una real influencia, mientras que los principiantes mantienen una reducida red de relaciones, aunque enriquecedoras y significativas con miembros principalmente de la asignatura.

Las relaciones con los colegas en el departamento o en el contexto universitario más amplio estarían limitadas a los principiantes y serían establecidas únicamente por los docentes de más experiencia, quienes toman la mayoría de las decisiones de la tarea. 
Revista de la Escuela de Ciencias de la Educación, año 11, número 10, enero a diciembre de 2015. PÁginas 131-148. ISSN 1851-6297. ISSN EN LINEA 2362-3349. RECONSTRUYENDO EL CONOCIMIENTO DIDÁCTICO DE PROFESORES UNIVERSITARIOS de la Universidad Nacional del Nordeste. Patricia B. Demuth

En este sentido, entendimos que el departamento en sí no se conformaría como el espacio de contención de los que se están iniciando, en un espacio de acogida o de acompañamiento, sino, que son determinadas personas, generalmente miembros de la propia asignatura pero también "otras" muy cercanas, las que aportan, guían, asesoran, o imponen conocimientos y prácticas.

Se nos planteaba pensar en este sentido, que los principiantes, en gran medida, tomarían los conocimientos didácticos de esos "otros", ya sea porque les resultaron positivos en su propio aprendizaje, o interesantes personalmente, o porque les eran impuestos. Cualquiera sea la circunstancia, parecía ser que los principiantes ponían a prueba, al menos, parte de los CDC de los otros y los hacían suyos, brindándoles singularidad y conformándolos de acuerdo a las características personales, y a las respuestas que iban obteniendo de sus estudiantes. Ese contexto educativo concreto también era fuente de ese nuevo conocimiento didáctico que se había visto transformado por el principiante.

En el caso de los experimentados, esa etapa de nutrirse principalmente de otros ya había quedado atrás, ahora primaban la propia experiencia en el transcurrir de los años, los criterios personales y profesionales, y la colaboración y el contacto con otros colegas, pero desde relaciones de igualdad y consulta para la adecuación al contexto concreto.

En ambas etapas podemos observar que el CDC de los profesores evidencia el solapamiento producido entre lo disciplinar, lo pedagógico y lo contextual (Escudero Escorza, 2003).

Claro que lo pedagógico, como ya dijimos, en la mayoría de los casos se centraba en un conocimiento práctico, aprendido del ejercicio profesional propio o de otros profesores más experimentados. Ahora bien, consideramos que esto no implicaría que ese conocimiento pedagógico del experimentado no sea en parte formal, pero sí es claro que su vinculación con estos principiantes ha sido a través del aprendizaje informal fruto de las relaciones interpersonales, que confirmaban la naturaleza eminentemente social de la cognición (Putnam y Borko, 2000), y el estado pre-profesional de la docencia de los segundos (Cruz Tomé, 2003).

Este proceso de apropiación que hicieran los principiantes de esos conocimientos que son compartidos y aprendidos a través de los otros que les rodean (Mayor Ruiz, 2008), más allá del contenido de lo aprendido, hayan sido mejores esquemas explicativos, estrategias de enseñanza, criterios o instrumentos de evaluación, más allá de ello, todo fue puesto a prueba con sus estudiantes, y la importante presencia de relatos que manifestaban opiniones, conocimientos o preocupaciones para con los aprendizajes, tenía que ver con esta puesta a prueba constante de lo aprendido informalmente y de aquello que proponían a partir de sus propias construcciones.

Observábamos que los principiantes entrevistados se encontraban en una etapa de "reconocimiento" de ese otro educativo que es el estudiante. Ellos 
Revista de la Escuela de Ciencias de la Educación, año 11, número 10, enero a diciembre de 2015. Páginas 131-148. ISSN 1851-6297. ISSN EN LINEA 2362-3349. RECONSTRUYENDO EL CONOCIMIENTO DIDÁCTICO DE PROFESORES UNIVERSITARIOS de la Universidad Nacional del Nordeste. Patricia B. Demuth

manifestaban haberlos observado, haber registrado lo sucedido, haber tenido presente sus intereses y estado atentos a sus logros y fracasos.

Etapa de "reconocimiento" en la que como docentes desconocían más que conocían de ese otro y que, por lo tanto, les demandaba tanta atención. Intentaban comprender lo que sucedía en sus salas de clase, estaban desarrollando su conocimiento docente (Oosterheert y Vermunt, 2003), y construyendo sus, cada vez más, adaptadas respuestas a los problemas de enseñanza y aprendizaje de la disciplina.

La construcción de mejores respuestas, les fue reportando mayor seguridad en los procesos de gestión de la clase y los fue ubicando en el rol de profesores, más allá de la cercanía de edad con sus estudiantes (Ávalos, 2007; Oplatka, 2007).

Podríamos decir entonces, que nuestros principiantes estaban en camino de convertirse en profesores autónomos (Vonk 1996 en Marcelo, 2007), pero quisiéramos puntualizar que en el ámbito universitario, esa autonomía no sólo tiene que ver con la "madurez" del conocimiento profesional del docente, sino también, con las posibilidades que le brinda este contexto institucional para ejercerla. Posibilidades, que en la universidad se otorgan, básicamente, en función de la jerarquía en el cargo que se desempeñe el profesor (Feixas, 2002), y que hasta el momento de las entrevistas habían sido muy escasas para los principiantes y muy formativas para los profesores con mayor experiencia. Que a diferencia de los principiantes, ya se han apropiado de dichos conocimientos docentes, elaborando teorías pedagógicas concretas a través de la puesta a prueba de sus actuaciones a lo largo de los años. En sus relatos pudimos observar que los experimentados tienen cabal conocimiento de los estudiantes y sus características, identifican claramente un número importante de obstáculos recurrentes para el aprendizaje de los contenidos de sus asignaturas y las posibles vías de acción para resolverlos.

Toman decisiones respecto de la gestión de las clases de manera fundamentada, con desarrolladas habilidades de interpretación para las diferentes situaciones del aula (Cf. Angulo Rasco, 2001).

En todos los casos son profesores autónomos potenciados por las funciones que les permite el ámbito universitario (como en el caso de los profesores adjuntos), o su rol en el equipo docente (como el caso de los profesores de trabajos prácticos de odontología).

Ahora bien, a partir de lo desarrollado ¿Cómo estaría constituida la identidad de los profesores universitarios? ¿Habría diferencias entre los casos estudiados? ¿Con qué aspectos o dimensiones se podrían plantear esas diferencias?

En nuestros intereses habíamos planteado indagar sobre las construcciones identitarias que podíamos inferir a partir de lo relevado. Identificarlos como colectivo profesional, distinguirlos del resto e ir develando aquello que los cohesionaba. Para responder a estas cuestiones tuvimos que esperar a 
Revista de la Escuela de Ciencias de la Educación, año 11, número 10, enero a diciembre de 2015. Páginas 131-148. ISSN 1851-6297. ISSN EN LINEA 2362-3349. RECONSTRUYENDO EL CONOCIMIENTO DIDÁCTICO DE PROFESORES UNIVERSITARIOS de la Universidad Nacional del Nordeste. Patricia B. Demuth

terminar todas las entrevistas y observaciones, a escapar de las impresiones individuales que los sujetos nos generaron, ya que no nos interesaba su identidad personal; y contemplar sus discursos como un todo, para profundizar en la identidad profesional que de éstos podría interpretarse. Nos interesaba qué estaban diciendo de ellos mismos y de los otros, cómo se reconocían, se auto-referenciaban.

En primer lugar, no pudimos en el caso total, identificar una identidad colectiva claramente homogénea del profesor universitario. Sólo rasgos comunes que "abren camino" al momento de hablar de identidades compartidas en los casos incrustados.

Hemos podido constatar una y otra vez, que la identidad de los grupos de profesores se orientaba hacia alguna de las dimensiones, en algunos casos pasaba por la dimensión Profesional, ellos eran, antes que nada: Informáticos u Odontólogos; en otro caso por la dimensión de Investigación: eran Historiadores, y en el último caso una singular mezcla de docencia e investigación: se reconocían como "cientistas de la educación".

De manera contundente, tanto en experimentados como en principiantes se planteaba de modo homogéneo al interior de los casos incrustados una identificación con algunos de los aspectos mencionados.

Dejando de lado a ciencias de la educación, nos parecía hasta de sentido común que los demás principiantes sólo hayan podido verse como informático, o como odontóloga o historiador, ya que los demás ámbitos que no reconocían, o estaban inexistentes o en sus inicios. Al entender que aprender a enseñar, ya sea por formación o por ejercicio, tendría que ver entonces con la construcción de la identidad de un profesor, en un nivel y disciplina determinados (Montero, 2001), cómo esperar que en este momento de su etapa profesional, pudieran identificarse con la docencia. En el caso de informática también se suma el contexto laboral en el nivel universitario argentino, que no podemos desconocer. La dedicación (en horas y retribución económica) es escasa para la gran mayoría de los profesores. Por lo tanto, si tenemos en cuenta que la menor carga horaria laboral de ellos estaba en el desempeño de la docencia, y su mayor carga, en el hacer informática, adquiría absoluto sentido que sus identificaciones fueran con las de la práctica como informáticos.

En el caso del principiante de historia, si bien su dedicación a la universidad es de tiempo completo, su mayor carga en la distribución horaria estaba puesta en la investigación, opción respaldada por la normativa institucional.

Y en el caso de la profesora principiante de odontología, su cultura profesional la condicionaba a mantener una práctica clínica por fuera de la academia más allá de la dedicación en la universidad, mostrando la fuerza que tiene la profesión independiente en el desarrollo profesional de los profesores de odontología. 
Revista de la Escuela de Ciencias de la Educación, año 11, número 10, enero a diciembre de 2015. Páginas 131-148. ISSN 1851-6297. ISSN EN LINEA 2362-3349. RECONSTRUYENDO EL CONOCIMIENTO DIDÁCTICO DE PROFESORES UNIVERSITARIOS de la Universidad Nacional del Nordeste. Patricia B. Demuth

En el caso de la profesora de informática con más experiencia, pudimos observar similares consideraciones a las de sus profesores principiantes, aunque su conocimiento docente se encontraba más desarrollado y fortalecido.

El caso de los profesores de odontología y de la profesora de historia con más experiencia, nos hizo pensar seriamente en el impacto de la formación docente de grado y de posgrado y su vinculación con las disciplinas concretas. Reflexión que retomaremos en las conclusiones.

Todo esto nos permitió plantear que la identidad de los sujetos se construía por la implicación de los mismos en las comunidades de práctica a las que pertenecían, y era influida e influyente en estas comunidades (Wenger, 2001), con lo cual podríamos en algunos casos hacer extensivo este planteo, sólo como supuesto, a todos los departamentos, en la medida que los relatos de los profesores lo habilite; y permitirnos pensar que la identidad de estos miembros está asentada en comunidades de práctica específicas que trascienden las características de la institución educativa en la que se desempeñan. $O$ acaso sería posible una conjunción de muchas identificaciones (fuera y dentro del ámbito laboral), unificadas en sus potencialidades, características y personajes (Bernal, 2003).

La identidad profesional de los profesores, trascendería entonces, sus entornos institucionales específicos y se mantendría fuerte, aún en un contexto institucional educativo. Contexto que dadas las características laborales que plantea, estaría limitando en la actualidad la apropiación de un proyecto profesional-institucional por parte de los principiantes.

Por otro lado, si en toda identidad profesional también interviene, por un lado, el auto-reconocimiento de un alto nivel de habilitación en el oficio (Ávila y Cortés, 2007), podíamos interpretar que nuestros profesores, reconocían una menor habilidad en la docencia y un alto nivel en la práctica como profesionales por fuera de la universidad o como investigadores. Sólo en el caso de ciencias de la educación este planteo es diferente, ya que la docencia atraviesa el campo de investigación y se asimila al de la profesión independiente de la universidad.

Otro factor que interviene como aspecto indispensable en la identidad profesional, es el reconocimiento social de la labor, que nos llevaba a preguntarnos, aunque no hemos podido interpretar respuestas para esto, si detrás de esta identificación exclusiva con la dimensión profesional o de investigación, no estarían influyendo también imaginarios sociales, en relación con el estatus de la docencia. Que también, en el ámbito universitario se ubica en el último nivel de consideración. Ya que el profesorado universitario se caracterizaba por tres funciones: la docente, la investigación y la gestión, y nosotros ya habíamos descartado la gestión por no estar vinculada con la docencia, desde las voces de nuestros protagonistas, y habíamos incluido la profesión como ámbito estrechamente relacionado al conocimiento profesional docente; pudimos detallar que los profesores en estudio ejercían, en el contexto universitario, el rol de 
Revista de la Escuela de Ciencias de la Educación, año 11, número 10, enero a diciembre de 2015. Páginas 131-148. ISSN 1851-6297. ISSN EN LINEA 2362-3349. RECONSTRUYENDO EL CONOCIMIENTO DIDÁCTICO DE PROFESORES UNIVERSITARIOS de la Universidad Nacional del Nordeste. Patricia B. Demuth

docentes, claro que, completamente atravesados por el rol profesional o de investigación.

Si, además, podemos ligar a la docencia y a la investigación, y sostener que la mayor o menor cercanía entre ambas tenía relación con las características epistemológicas del campo disciplinar; nos cuestionamos si esta ausencia o escasa presencia de la dimensión de investigación en los casos de Informática y Odontología, se debía a esas características disciplinares o a las condiciones contextuales institucionales y locales; o a ambas, ya que podíamos suponer, según lo relevado, que la investigación en informática y Odontología demandaría otras condiciones de desarrollo en el ámbito en el que se la realice.

Respecto de Historia, podemos reflexionar cómo la tarea del historiador que es la investigación, se ubica por encima de la del profesor, que es la de enseñar historia, dando cuenta de la primacía de la primera sobre la segunda.

Y en el caso de Ciencias de la Educación la cercanía entre docencia e investigación se vuelve entrecruzamiento, dado el solapamiento de las actividades de investigación sobre los mismos fenómenos en los cuales se inserta también la actividad docente.

Planteadas así las cuestiones, pudimos observar que la comprensión de las dimensiones, especialmente, la comprensión del entramado sobre el conocimiento docente generado a partir de los relatos, requería necesariamente de los detalles del contexto, de otros profesores, de su formación, de sus intereses y propuestas, detalles que tomados aisladamente parecían no tener relevancia para los sujetos que investigábamos. Sin embargo, la posibilidad de entender de manera más acabada lo que sucedía con ellos y su conocimiento profesional, la posibilidad de profundizar en algunas explicaciones; esa posibilidad se inscribía ineludiblemente en el contexto del que formaban parte, en las conexiones y vínculos que con él se establecían, en las mutuas referencias que brindaban sentido a los procesos de enseñar y de aprender que estaban vivenciando estos sujetos.

\section{A modo de cierre...}

Para ir finalizando este relato, retomamos la necesidad de reconocer la asignatura y su contexto, en primer lugar, y del departamento, en segundo lugar, como espacios en los que se generan las relaciones de mayor influencia para el proceso de "aprender a enseñar" de un principiante y de "enseñar a enseñar" de un experimentado. $Y$ a partir de este reconocimiento el planteo de la necesidad de incorporación de los principiantes a las tareas de reflexión, diseño y elaboración de los programas de la materia y de los planes de estudio de la carrera. Actividad que repercutiría positivamente en el desarrollo y la reflexión sobre el propio conocimiento disciplinar en un sentido íntegro. 
Revista de la Escuela de Ciencias de la Educación, año 11, número 10, enero a diciembre de 2015. Páginas 131-148. ISSN 1851-6297. ISSN EN LINEA 2362-3349. RECONSTRUYENDO EL CONOCIMIENTO DIDÁCTICO DE PROFESORES UNIVERSITARIOS de la Universidad Nacional del Nordeste. Patricia B. Demuth

A su vez, nos ponemos a pensar sobre lo poderosa que es la herramienta de la narración como medio de reflexión sobre una determinada 'realidad', reflexión que nos llevó a una mejor comprensión de lo relevado, a la posibilidad de realizar interacciones precisas con los marcos referenciales (que siempre estuvieron presentes), debido a que nos permitió repensar la noción conocimiento didáctico del contenido, principalmente a sus componentes: ya que si bien el CDC de estos profesores tiene particularidades, resultó innegable la fuerza y presencia que tiene la disciplina, tanto desde su dimensión académica como su dimensión práctica y contextual. Situación que movilizó sustancialmente nuestras jerarquías categoriales y supuestos, y que nos hace reflexionar que si al estar más débiles algunas dimensiones docentes, a los profesores sólo les resta transformar su experiencia laboral (como práctica y conocimiento del hacer en su disciplina) o su experiencia de investigación, en representaciones didácticamente enseñables. Y un trabajo comprensivo como el planteado podría guiar algunas líneas de acción para el desarrollo profesional docente desde la inserción misma en la carrera docente universitaria.

\section{Referencias Bibliográficas}

- $\quad$ Angulo Rasco, J. (1999). "De la investigación sobre la enseñanza al conocimiento docente" en Pérez Gómez, A. I. ; Barquín Ruiz, J. \& Angulo Rasco, J. F. Desarrollo Profesional del Docente. Política investigación y Práctica (261-319). Madrid: Akal.

- Ávalos, B. \& Aylwin,P. (2007). How young teachers experience their professional work in ChileTeaching and Teacher Education,23, 515-528.

- Ávila Quevedo, J. A. \& Cortés Montalvo,J.(2007). La construcción de las identidades profesionales a través de la educación superior Cognición(9).

- Bain, K. (2007). Lo que hacen los mejores profesores universitarios. Valencia: Publicacions Universitat de Valencia.

- Bernal Guerrero, A. (2003). La construcción de la identidad personal como proyecto de educación moral: supuestos teóricos y delimitación de competencias. Teoría de la educación, 15, 129-160.

- Bolívar, A. (2005). "Conocimiento didáctico del contenido y didácticas específicas". En Profesorado. Revista de currículum y formación del profesorado, 9, 1-39.En Profesorado. Revista de curriculum y formación del profesorado 9.

- Becher, T. (2001). Tribus y Territorios Académicos. La indagación intelectual y las culturas de las disciplinas. Barcelona: Gedisa.

- $\quad$ Cid-Sabucedo, A., Pérez-Abellás, A., \& Zabalza, M. (2009). Las prácticas de enseñanza declaradas de los "mejores profesores" de la Universidad de Vigo. Relieve, 15(2), 1-29.

- Cubero, R. (2005). Perspectivas Constructivistas. La intersección entre el significado, la interacción y el discurso. Barcelona: Graō.

- $\quad$ Cruz Tomé, M. (2003). Necesidad y objetivos de la formación pedagógica del profesor universitario. Revista de Educación, 331,35-66.

- $\quad$ Elbaz, F. (1983). Teacher thinking: A study of practical knowledge.London: Croom Helm.

- Feixas, M. (2002). El desenvolupament profesional del profesor universitari com a docent. Tesis Doctoral. Departamento de Pedagogía Aplicada, Universidad Autónoma de Barcelona, Barcelona, España. 
Revista de la Escuela de Ciencias de la Educación, año 11, número 10, enero a diciembre de 2015. Páginas 131-148. ISSN 1851-6297. ISSN EN LINEA 2362-3349. RECONSTRUYENDO EL CONOCIMIENTO DIDÁCTICO DE PROFESORES UNIVERSITARIOS de la Universidad Nacional del Nordeste. Patricia B. Demuth

- Fourez, G. (2008). Cómo se elabora el conocimiento. La epistemología desde un enfoque socio constructivista. Madrid: Narcea.

- $\quad$ Grossman, P. (1990). The making of a teacher: teacher knowledge and teacher education. New York: Teachers College Press.

- Gudmundsdottir, S. (1990). Curriculum stories: Four case studies of social studies teaching. Insights into teachers' thinking and practice, 107-118.

- Jiménez-Chaves V.E. (2012) El estudio de caso y su implementación en la investigación. Rev. Int. Investig. Cienc. Soc., Vol. 8, (1), pp. 141-150.

- Marcelo, C. (2006). La formación docente en la sociedad del conocimiento y la información: avances y temas pendientes. Ponencia en el Cuarto Encuentro Internacional de KIPUS. Políticas públicas y formación docente, Isla Margarita, Venezuela.

- Marcelo, C. (2007). Empezar con buen pie: inserción a la enseñanza para profesores principiantes. Docencia, 33,27-38.

- $\quad$ Mayor Ruiz, C. (2007). El asesoramiento pedagógico para la formación docente del profesorado universitario.Sevilla, Secretariado de publicaciones de la Universidad de Sevilla.

- Montero, L. (2001). La construcción del conocimiento profesional docente. Rosario: Homo Sapiens.

- Neiman, G; Quaranta, G. (2006) "Los estudios de caso en la investigación sociológica”. En: Vasilachis de Gialdino (comp.) Estrategias de investigación cualitativa. Buenos Aires: Gedisa

- $\quad$ Oosterheert, I.\& Vermunt J. (2003). Knowledge Construction in Learning to Teach: the role of dynamic sources. Teachers and Teaching, 9, 157 - 173.

- $\quad$ Oplatka, I., \& Eizenberg, M. (2007). The perceived significance of the supervisor, the assistant, and parents for career development of beginning kindergarten teachers. Teaching and Teacher Education, 23(4), 339-354.

- $\quad$ Putnam, R. T., \& Borko, H. (2000). "El aprendizaje del profesor: implicaciones de las nuevas perspectivas de la cognición”. En: B. J. Biddle, T. L. Good, y I. F. Goodson (Eds.) La enseñanza de los profesores I. Barcelona, España: Paidos

- Rodríguez Rojo, M. (2000). Credibilidad de la investigación cualitativa o reflexiones sobre qué y cómo observar en un estudio de casos. Revista interuniversitaria de formación del profesorado, № 39 , pp. 119-130

- Schön, D. (1992). La formación de profesionales reflexivos. Barcelona: Paidós.

- $\quad$ Stake, R. (2007) Investigación con estudios de caso. Madrid: Editorial Morata.

- Wenger, E. (2001). Comunidades de práctica. Aprendizaje, significado e identidad. Barcelona: Paidós. 